\title{
CCR6+ group 3 innate lymphoid cells accumulate in inflamed joints in rheumatoid arthritis and produce Th17 cytokines
}

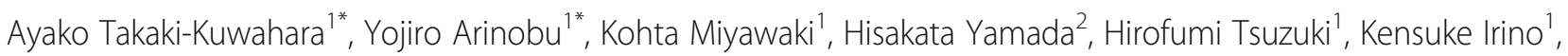
Masahiro Ayano ${ }^{3}$, Yasutaka Kimoto ${ }^{4}$, Hiroki Mitoma ${ }^{1}$, Mitsuteru Akahoshi ${ }^{1}$, Hiroshi Tsukamoto ${ }^{1}$, Takahiko Horiuchi ${ }^{4}$, Hiroaki Niiro ${ }^{5}$ and Koichi Akashi ${ }^{1}$

\begin{abstract}
Background: Recent studies show that innate lymphoid cells (ILCS) contribute to the development of chronic inflammation and autoimmune disease. In this study, we assessed the ILC function in the development of rheumatoid arthritis (RA).
\end{abstract}

Methods: In a mouse model of collagen-induced arthritis (CIA), we identified and purified the ILC subsets in peripheral blood (PB), local lymph nodes (LNs), and joints by fluorescence-activated cell sorting and used quantitative PCR to assess the expression levels of representative cytokines. We also correlated the frequencies of each ILC subset in synovial fluid (SF) with clinical parameters in RA patients.

Results: In the CIA model, the proportion of CCR6+ ILC3s to total ILCS in joints with active inflammation significantly increased relative to non-arthritic joints (median $29.6 \%$ vs $16.7 \%, p=0.035$ ). CCR6+ ILC3s from mice with arthritis expressed significantly higher levels of IL-17A and IL-22 mRNA than did comparable cells from control mice $(p<0.0001$ and $p=0.015)$. In RA patients, the proportion of CCR6+ ILCs in SF was positively correlated with tender joint counts (TJC) and swollen joint counts (SJC) ( $\rho=0.689, p=0.0032$ and $\rho=0.644, p=0.0071$, respectively). Levels of CC chemokine ligand 20 (CCL20) increased in SF of patients with RA and were significantly correlated with CCR6+ ILC number ( $\rho=$ $0.697, p=0.0001)$.

Conclusion: CCR6+ ILC3s may play some roles in the development of RA through the production of IL-17 and IL-22.

Keywords: Innate lymphoid cells, Rheumatoid arthritis, CCR6, Th17 cytokines

\section{Background}

Innate lymphoid cells (ILCs) are recently discovered immune cells that do not express rearranged antigen receptors and do not have antigen specificity [1]. ILCs represent the innate immune system's counterpart to helper $\mathrm{T}$ cells and are classified into three subsets, group 1 (ILC1s), group 2 (ILC2s), and group 3 (ILC3s), based

\footnotetext{
* Correspondence: t-ayako@med.kyushu-u.ac.jp;

yarinobu@cancer.med.kyushu-u.ac.jp

${ }^{1}$ Department of Medicine and Biosystemic Science, Kyushu University Graduate School of Medical Sciences, 3-1-1 Maidashi, Higashi-ku, Fukuoka 812-8582, Japan

Full list of author information is available at the end of the article
}

on the patterns of cytokine production and transcription factor expression [1]. Accordingly, ILC1s require T-box transcription factor (T-bet) for differentiation and produce interferon- $\gamma$ (IFN- $\gamma$ ); ILC2s express the highest levels of GATA-binding protein 3 (GATA-3) and produce interleukin-4 (IL-4), IL-5, IL-9, and IL-13; and ILC3s express and are regulated by retinoic acid receptor-related orphan receptor $\gamma \mathrm{t}(\mathrm{ROR} \gamma \mathrm{t})$ and produce IL17 and IL-22. ILC3s are further subdivided based on the expression of either CC chemokine receptor 6 (CCR6) or natural cytotoxicity receptor (NCR), which is constituted by NKp46, NKp44, and NKp30 and is expressed mainly on natural killer (NK) cells [2, 3]. Increasing

(C) The Author(s). 2019 Open Access This article is distributed under the terms of the Creative Commons Attribution 4.0 International License (http://creativecommons.org/licenses/by/4.0/), which permits unrestricted use, distribution, and 
evidence shows that ILCs contribute to chronic inflammation and autoimmune disease [4-6]. There are some reports that show ILC3s are the key population in inflammation of spondyloarthritis (SpA) [7-10]; on the other hand, a limited number of studies have addressed the role of ILCs in rheumatoid arthritis (RA), and it is controversial which subset is important in RA development. Recent studies revealed that ILC2s are essential for the resolution of inflammation in RA [11, 12], although another group showed that GM-CSF-producing ILC2s play a role in the development of arthritis [13]. In contrast, ILC1-like and ILC3-like cells have been shown to promote inflammation in RA [14-16]. ILC3s produce IL-17 and IL-22, cytokines reportedly essential for RA pathogenesis $[7,8,17]$. Furthermore, CCR6 has been identified as an RA disease susceptibility gene [18]. These findings suggest overall that CCR6+ ILC3s function in RA development.

In this study, we evaluated the number, distribution, and function of ILC1, ILC2, and ILC3 cells in a murine arthritis model and in patients with RA. Our results suggest that CCR6+ ILC3s play a crucial role in RA development.

\section{Materials and methods}

Mice

Male C57BL/6J mice were evaluated at 11-12 weeks of age. All mice used in this experiment were purchased from Charles River Japan Inc. (Yokohama, Japan) and maintained under specific pathogen-free conditions at the Kyushu University Animal Facility. All animal experiments were approved by the Animal Care and Use Committee, Kyushu University.

\section{Induction of collagen-induced arthritis}

Mice were immunized subcutaneously at the base of the tail with $4 \mathrm{mg} / \mathrm{ml}$ chicken type II collagen (CII) (Collagen Research Center, Tokyo, Japan) emulsified with an equal volume of complete Freund's adjuvant (CFA) containing Mycobacterium tuberculosis H37Ra at a concentration of $5 \mathrm{mg} / \mathrm{ml}$ (Difco, Detroit, MI, USA). Mice were boosted on day 21 with CII emulsified with CFA containing Mycobacterium tuberculosis H37RA at the same concentration.

\section{Measurement of serum anti-type II collagen antibody levels}

Serum levels of anti-CII antibodies were measured by enzyme-linked immunosorbent assay (ELISA). Microtiter plates were coated with chicken CII $(10 \mu \mathrm{g} / \mathrm{ml})$ overnight at $4{ }^{\circ} \mathrm{C}$. After washing and blocking, serum samples were added in serial dilutions and incubated $2 \mathrm{~h}$ at room temperature. After four washes, HRP-conjugated antimouse IgG antibody (Leinco Technologies Inc., St. Louis, MO, USA) was added and incubated for an hour at room temperature. Antibody binding was visualized using TMB substrate (Seracare Life Sciences Inc., Milford, MA, USA). All plates were read at $450 \mathrm{~nm}$ using MultiSkan FC (Thermo Fisher Scientific KK, Yokohama, Japan).

\section{Flow cytometric analysis of mouse ILC subsets}

To obtain a cell suspension from mouse joints, the entire legs were dissected and the muscles and tendons were removed. To avoid contamination with the bone marrow, the femur was disarticulated by pulling the femoral head. The ligaments and tendons around the joints were cut a few millimeters. The knee and ankle joint were then opened. The legs were incubated in a mixture of enzymes containing $100 \mathrm{U} / \mathrm{ml}$ collagenase (Wako Pure Chemical Industries, Ltd., Osaka, Japan) and $100 \mu \mathrm{g} / \mathrm{ml}$ DNaseI (Sigma-Aldrich, St. Louis, MO, USA) and shaken for $60 \mathrm{~min}$ at $37^{\circ} \mathrm{C}$. After digestion, the legs were removed, and cells were purified using Percoll density centrifugation, filtered, and counted using automated cell counter.

The popliteal lymph nodes and spleen were mechanically mashed and filtered. Splenocytes and peripheral blood cells were hemolyzed with $\mathrm{NH}_{4} \mathrm{Cl}$ and washed with PBS. For surface staining, single-cell suspensions of joint cells, lymph node cells, splenocytes, and peripheral blood cells were washed with cell staining buffer and stained with a cocktail of antibodies for $30 \mathrm{~min}$ at $4{ }^{\circ} \mathrm{C}$. ILCs were defined as CD45.2 positive, lineage (CD3, CD8, CD19, B220, Ter119, Ly-6G, CD11c, CD11b) negative, CD90.2 (Thy1.2) positive, and CD127 (IL-7R) positive. ILCs were subsequently classified as ILC1s, ILC2s, CCR6+ ILC3s, or NKp46 (CD335)-positive ILCs (NKp46+ ILCs), based on the expression of NK1.1, ST2 (the receptor for IL-33), CCR6, and NKp46.

\section{RNA extraction and quantitative real-time PCR}

Each target population was sorted directly into ISOGEN II (Nippon Gene Co., Tokyo, Japan), and total RNA was extracted and reverse transcribed using the SuperScript III First-Strand Synthesis System (Invitrogen). Quantitative real-time PCR was conducted with a 7500 RealTime PCR System (Applied Biosystems, Foster, CA, USA) or an Mx 3000P Real-Time PCR System (Agilent Technologies, Santa Clara, CA, USA). TaqMan probes for Tbx21 (Mm00450960_m1), Gata3 (Mm00484683 $\mathrm{m} 1)$, RORC (Mm01261022_m1), Ifng (Mm01168134_ m1), Il5 (Mm00439646_m1), Il13 (Mm99999190_m1), Il17Aa (Mm00439618_m1), and Il22 (Mm00444241_m1) were all purchased from Applied Biosystems. 18S rRNA expression served as the internal control.

Relative transcript levels were calculated using the ddCT method. 


\section{Patients}

Patients with RA met the 1987 American College of Rheumatology classification criteria. Twenty-three synovial fluid (SF) samples were obtained from 17 patients with RA. Patient details are provided in Additional file 8: Table S2. Twenty-four SF samples of patients with osteoarthritis (OA) were obtained from 20 patients. Informed consent was obtained from all subjects in accordance with the Declaration of Helsinki. The Institutional Review Board of Kyushu University Hospital approved all research on human subjects.

\section{Flow cytometric analysis of ILCs in synovial fluid in RA patients}

Mononuclear cells were isolated from SF using density gradient centrifugation with LSM (MP Biomedicals, LLC, Santa Ana, CA, USA). ILCs were defined as single cells within the lymphocyte gate on the scatter plot that were CD45 positive, lineage (CD3, CD19, CD16, CD94, CD14, CD1c, CD11b, CD11c, CD235ab, FceRI, CD34) negative, and CD127 (IL-7R) positive. ILC subsets were classified based on the expression of CD117 (c-Kit), chemoattractant receptor homologous molecule expressed on Th2 cells (CRTH2) (CD294), and NKp44 (CD336). Specifically, ILC2s were defined as CRTH2 positive, and other subsets were CRTH2 negative. ILC1s, NKp44- ILC3s, and NKp44+ ILC3s were defined as CD117- NKp44-, CD117+ NKp44-, and CD117+ NKp44+, respectively. CCR6+ ILCs were defined as CCR6-positive cells in total ILCs. Antibodies used in flow cytometric analysis are listed in Additional file 7: Table S1 and were purchased from BD Biosciences (San Jose, CA, USA), BioLegend (San Diego, CA, USA), Beckman Coulter (Villepinte, France), or MD Bioproducts (Saint Paul, MN, USA). Cells were analyzed with a FACS Aria III system (BD Biosciences). FlowJo software (Tree Star Inc., Ashland, OR, USA) was used to analyze flow cytometric data.

\section{Chemokine measurement}

SF from RA and OA patients was centrifuged, and supernatants were collected and stored at $-80^{\circ} \mathrm{C}$. Human CC chemokine ligand 20 (CCL20) in SF was measured by ELISA using a Human CCL20 ELISA kit (BioLegend) with a detection limit of $2.9 \mathrm{pg} / \mathrm{ml}$, according to the manufacturer's instructions.

\section{Statistical analysis}

Statistical comparisons between the two groups were performed using Student's $t$ test or the Wilcoxon ranksum test according to the distributions. Results are expressed as means \pm SD or the median (range). Differences between the three groups were assessed by the Kruskal-Wallis test followed by the Wilcoxon rank-sum test. Spearman's rank correlation was performed to correlate the proportion of ILC subsets in SF with clinical parameters of RA patients. $P$ values $<0.05$ were considered significant. All analyses were performed using JMP statistical software version 13.0 (SAS Institute, Cary, NC, USA).

\section{Results}

Identification of ILC1s, ILC2s, and ILC3s in wild-type mice We identified ILC subsets in splenocytes of adult male C57BL/6J mice. Identification of ILC1s, ILC2s, and ILC3s by surface markers $[1,19]$ is described in the "Materials and methods" section (Fig. 1a). ILCs were isolated as cells negative for lineage markers and positive for CD45, CD90, and CD127. ILCs were subsequently classified as CCR6+ ILC3s or NKp46+ ILCs. In cells negative for both CCR6 and NKp46, ILC1s and ILC2s were defined based on the expression of NK1.1 and ST2, respectively. In our gating strategy, NKp46+ ILCs include a part of ILC1s and NCR-positive ILC3s, as NKp46 is expressed on the surface of both. Because most NK cells express lineage marker CD11b and do not express CD127 [20], the majority of NK cells should not be included in ILC fraction.

We next confirmed the identity of each ILC subset using quantitative real-time PCR analyses to detect representative transcription factors (TFs) and cytokines in purified subpopulations (Fig. 1b, c). Expression levels of T-box 21 (Tbx21) and IFN- $\gamma$ mRNA increased in both ILC1s and NKp46+ ILCs. ILC2s expressed the highest level of GATA3 mRNA, while other ILCs expressed more moderate levels. IL-5 and IL-13 mRNAs were highly expressed in ILC2s, and CCR6+ ILC3s showed increased transcript levels of RAR-related orphan receptor C (RORC), IL-17A, and IL-22. These data indicate successful fractionation of ILC subsets.

\section{CCR6+ ILC3 populations expand in the joints of arthritic mice}

We next evaluated the ILC subsets in PB, popliteal lymph nodes (PLNs), and joints isolated from collageninduced arthritis (CIA) mice. Following CIA induction, mice exhibited both arthritic and non-arthritic phenotypes. However, although the latter group did not exhibit evidence of joint swelling, we detected anti-CII antibodies in their sera (Additional file 1: Figure S1), suggesting that these mice represent a preclinical phase of RA development. Thus, we analyzed these mice as "prearthritic mice." RA synovitis is initiated by an influx of leukocytes from the PB to the synovial tissue. Therefore, we asked whether the number of ILCs increased in inflamed joints of CIA mice, and if so, which subset accumulated in the joints. To do so, we first counted the absolute number of ILCs in the PB, PLNs, and joints of 


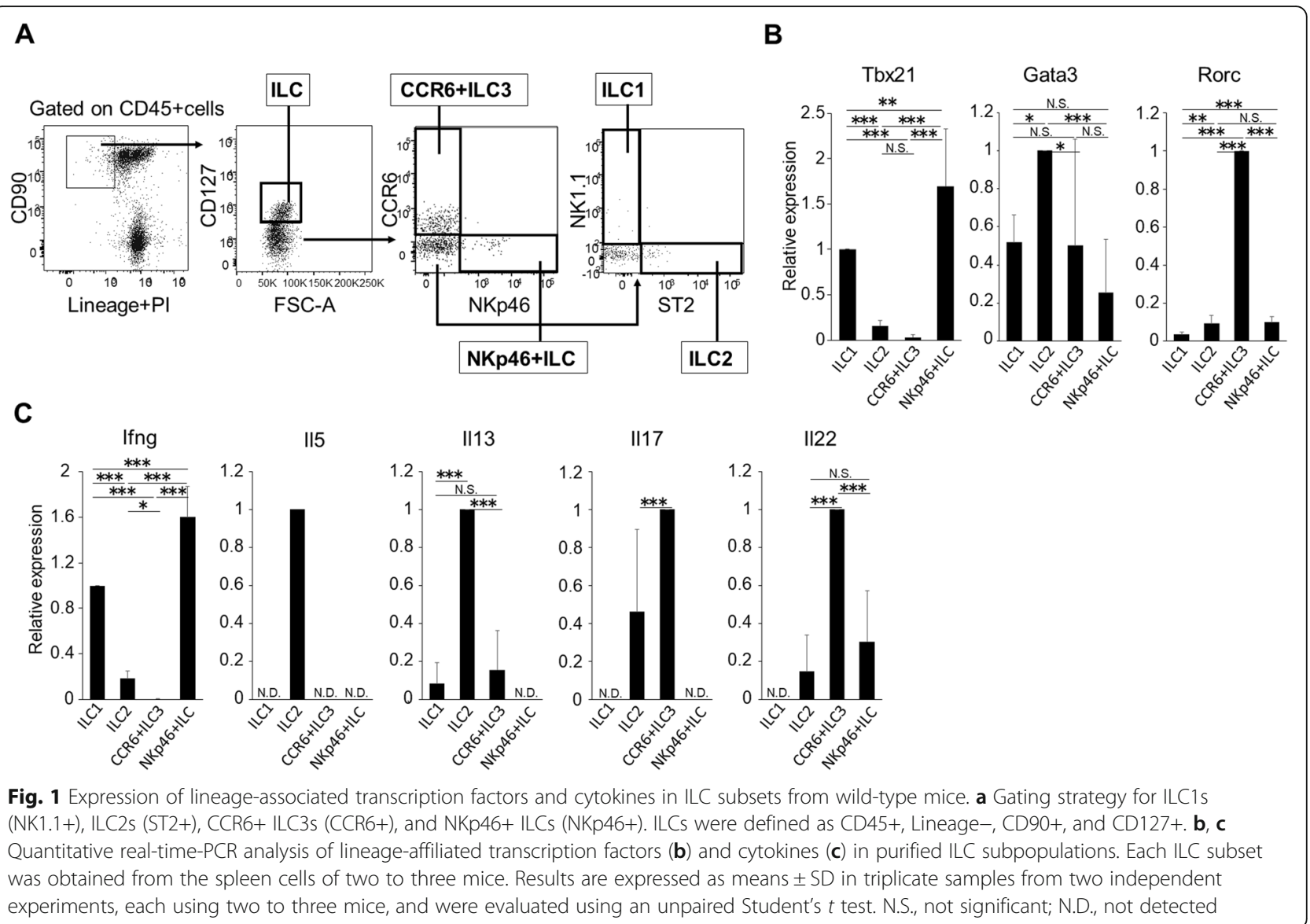

pre-arthritic, arthritic, and wild-type mice as control (Fig. 2a). We observed comparable numbers of ILCs in $\mathrm{PB}$ of mice in all three conditions. The number of ILCs in the PLNs of arthritic mice was higher than seen in control mice, although that increase was not statistically significant (median, $1.8 \times 10^{4} / \mathrm{lymph}$ node vs $8.8 \times 10^{3}$ / lymph node, $p=0.089$ ). ILC numbers increased in the joints of arthritic mice but were minimal in the joints of control mice. In short, the number of ILCs increased in local lymph nodes and joints of arthritic mice, although differences were not statistically significant. This result suggests that ILCs accumulate at the inflammatory sites.

We next calculated the ratios of ILC subsets to total ILCs in the joints of pre-arthritic and arthritic mice, while ILC number in the joints of control mice was too minimal to be analyzed. In arthritic mice, the proportion of CCR6+ ILC3s to total ILCs increased significantly compared to that seen in the joints of pre-arthritic mice (median, $29.6 \%$ vs $16.7 \% p=0.035$ ), while the proportions of other ILC subsets were comparable between the two groups (Fig. 2b). When we evaluated the CCR6+ ILC3/total ILC ratio in PB, PLNs, and joints of arthritic mice, we observed a higher relative ratio in $\mathrm{LN}$ than in PB $(18.6 \%$ vs $6.2 \%, p=0.00020)$ and a higher ratio in joints than in $\mathrm{LN}(29.6 \%$ vs $18.6 \%, p=0.057)$, although the latter was not statistically significant (Fig. 2c). We interpret this gradual change as due to the induction of severe arthritis, as we observed no differences in the proportion of CCR6+ ILC3s to total ILCs in the joints and LN of pre-arthritic mice $(16.7 \%$ vs $16.1 \%, p=0.58)$ (Additional file 2: Figure S2). These data indicate that CCR6+ ILC3s may preferentially accumulate at local sites of inflammation.

\section{CCR6+ ILC3s produce high levels of IL-17A and IL-22 in arthritic mice}

Next, we compared cytokine mRNA levels in ILC subpopulations isolated from the spleen of control and arthritic mice. CCR6+ ILC3s in arthritic mice exhibited significantly higher expression levels of IL-17A and IL22 mRNA relative to control mice (Fig. 3a). The level of IFN- $\gamma$ transcripts in ILC1s was comparable in control and CIA mice. The expression of IL-5 mRNA was confirmed only in ILC2 of control mice, and the expression level of IL-13 mRNA was lower in arthritic mice than in control mice (Fig. 3b). These results suggest that the increase in cytokine production in CIA mice is specific to CCR6+ ILC3s. 

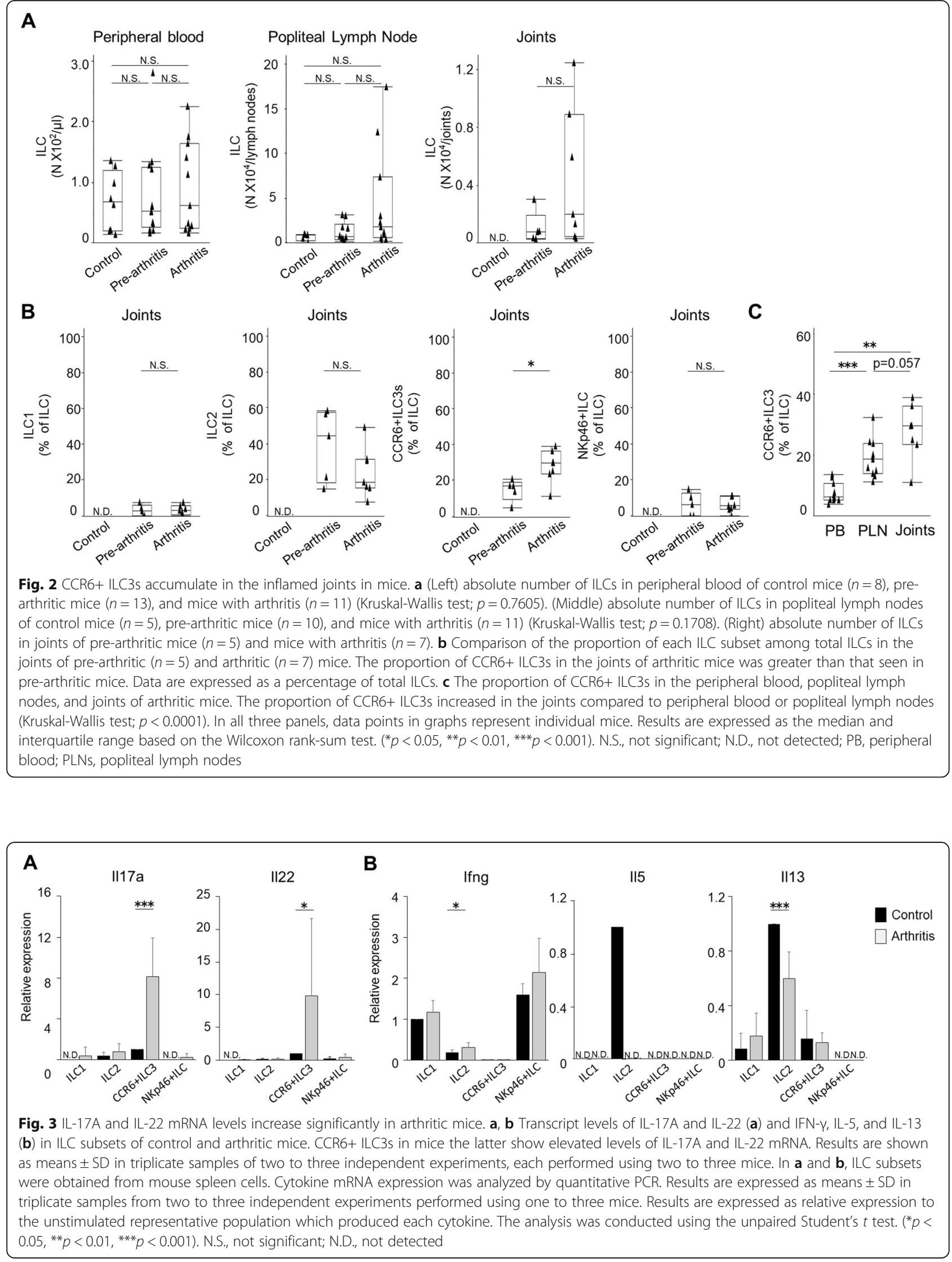
SF of patients with active RA shows increased numbers of CCR6+ ILCs

We next extended our analysis to human RA. To determine whether the proportion of CCR6+ ILCs to total ILCs increases in SF of RA patients as it does in the joints of CIA mice, we analyzed ILC subsets in 23 samples of RA SF. To examine whether CCR6 could be a marker of disease activity, we evaluate CCR6-positive cells in ILCs separately from the other ILC subsets (Fig. 4a). The other subsets, ILC1, ILC2, NKp44- ILC3, and NKp44+ ILC3, were evaluated using methods previously established [21] (Additional file 3: Figure S3A). CCR6+ ILCs in human peripheral blood express RORC, IL-17A, and IL-22 mRNA (Additional file 4: Figure S4) and produce these cytokines after stimulation (Additional file 5: Figure S5). The proportion of CCR6+ ILCs among total ILCs in SF of RA patients ranged widely from 5.36 to $83.6 \%$, while the proportions of other subsets in patients showed less variation (Fig. 4b).

To assess the characteristics of patients exhibiting a high proportion of CCR6+ ILCs, we examined the potential correlation of the proportion of each ILC subset in SF with clinical parameters by calculating Spearman's correlation coefficients. Clinical parameters assessed were age at examination, disease duration, swollen joint count (SJC), tender joint count (TJC), C-reactive protein $(\mathrm{CRP})$ levels $(\mathrm{mg} / \mathrm{dl})$, erythrocyte sedimentation rate (ESR) $(\mathrm{mm} / \mathrm{h})$, rheumatoid factor $(\mathrm{RF})$ levels $(\mathrm{U} / \mathrm{ml})$, and anti-CCP antibody levels (U/ml) (Additional file 9 Table S3). We excluded sex from the analysis, as there were only three male patients. We found that the proportion of CCR6+ ILCs increased with TJC $(\rho=0.689, p=0,0032)$ and with SJC $(\rho=0.644, p=0.0071)$ (Fig. 4c).

Relevant to other ILC subsets, ILC1 proportions were negatively correlated with TJC and SJC, while ILC2s numbers were not significantly correlated with clinical parameters. The proportion of NKp44+ ILC3s was positively correlated with TJC and SJC (Fig. 4d and Additional file 9: Table S3). The correlation of NKp44+ ILCs with SJC and TJC may reflect the expression of CCR6 protein on their surface because almost all NKp44+ ILC3s expressed CCR6 protein (Additional file 3: Figure S3B). Taken together, these results suggest that

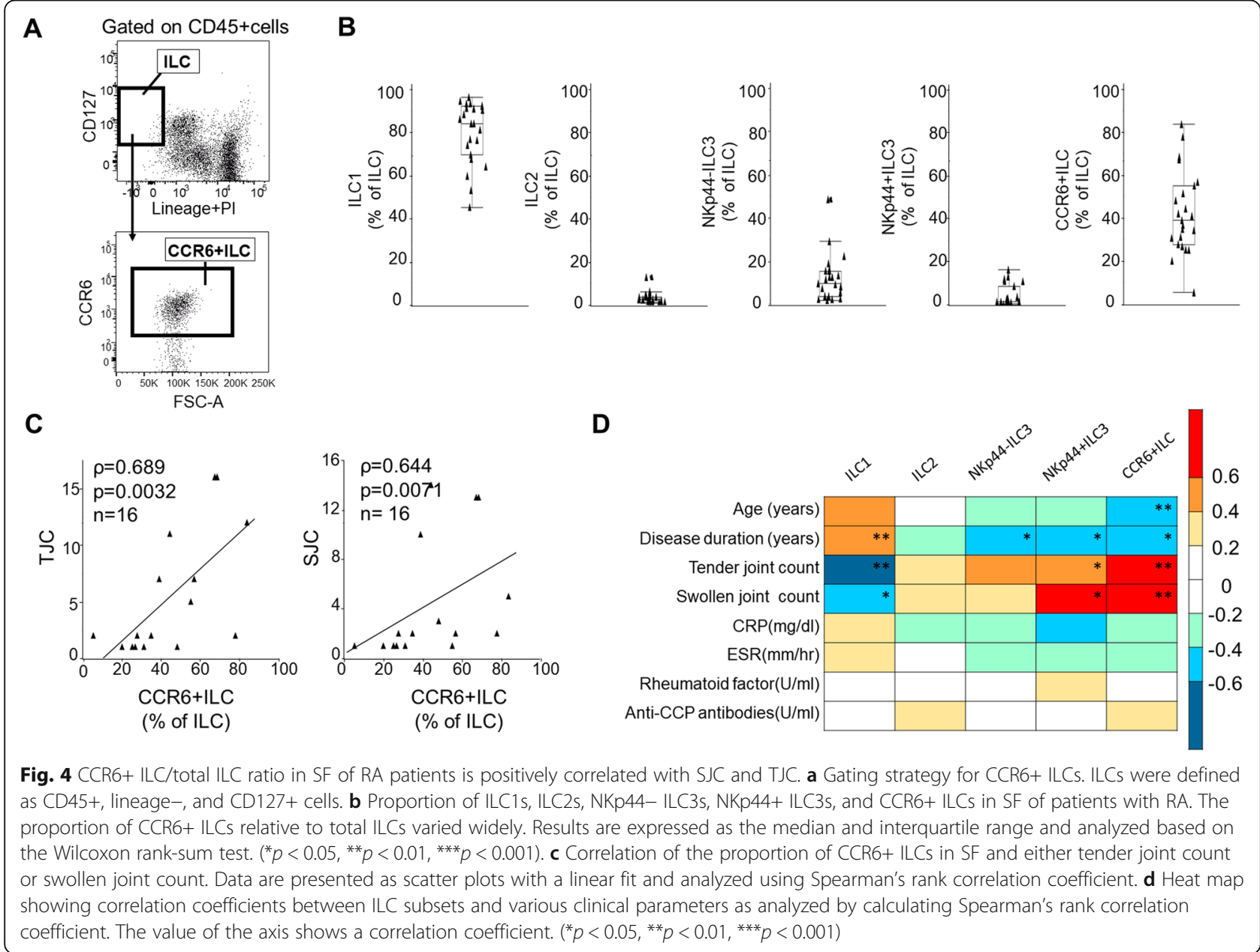


the presence of CCR6+ ILCs exacerbates joint inflammation in patients with RA.

\section{CCR6+ ILCs migrate to the joints in parallel with increasing CCL20 levels in SF of RA patients}

The CCR6 ligand CCL20 controls the migration of CCR6+ cells [22]. Thus, we evaluated CCL20 levels in SF of $14 \mathrm{RA}$ and in 13 control OA patients. The latter showed barely detectable CCL20 levels, while SF from RA patients showed high CCL20 levels, as previously reported [23] (Fig. 5a). Next, we examined the relationships between CCL20 concentration and absolute numbers of CCR6+ ILCs in SF. As shown in Fig. 5b, c, the absolute number of CCR6+ ILCs was significantly higher in SF of RA patients and CCL20 levels increased in parallel with the absolute number of CCR6+ ILCs.

\section{Discussion}

In this study, we evaluated the roles of ILCs in RA development using model mice and human patients. In CIA mice, CCR6+ ILC3s accumulated in the joints and exhibited high expression of mRNAs encoding the cytokines IL-17A and IL-22. In humans, the number of CCR6+ ILCs increased in SF of patients with active RA and accumulated in inflamed joints in parallel with CCL20 concentration in SF. These results suggest that CCR6+ ILC3s have some roles in the development of RA.

To date, there has been no consensus about which ILC subset has the greatest impact on RA progression. Hirota et al. showed that GM-CSF-producing ILC2s had a pathogenic role in the development of arthritis [13], although recent studies reported that ILC2s had a protective role $[11,12]$. Zhu et al. reported that the number of CD3- CD56+ NKp44+ cells, which are ILC3-like cells, increases in SF and PB of patients with RA and that those numbers are positively correlated with disease activity $[15,16]$. On the other hand, a subset of NK cells, which are ILC1-like cells, was identified in SF of patients with inflammatory arthritis including RA, spondyloarthropathy, and juvenile rheumatoid arthritis [14]. Leijten et al. also demonstrated that ILC1s predominate in RA SF [7], and Rodríguez-Carrio et al. revealed that the frequency of ILC1s in LN biopsy was significantly increased in RA patients [24]. However, in our hands, the ILC1 subpopulation did not increase in the joints of CIA model mice nor were Th1 cytokine expression levels elevated after arthritis induction. Furthermore, although ILC1s were the predominant population in SF of RA patients, their proportion to total ILCs was negatively correlated with the number of inflamed joints, suggesting that a different cell type is a critical factor in RA inflammation.

In this study, we observed differences in ILC phenotypes between mice and humans. The proportion of CCR6+ ILC3s in the joints of CIA mice increased in almost all cases, while there was considerable variability in the proportion of CCR6+ ILCs between SF samples from RA patients (Figs. $2 \mathrm{~b}$ and $4 \mathrm{~b}$ ). It is largely recognized that IL-17 signaling plays a key role in inflammatory arthritis in mouse models [25], while in humans, IL-17 signaling does not always play a critical role in inflammation associated with RA. Several anti-IL-17 therapies for RA have not demonstrated sufficient
A

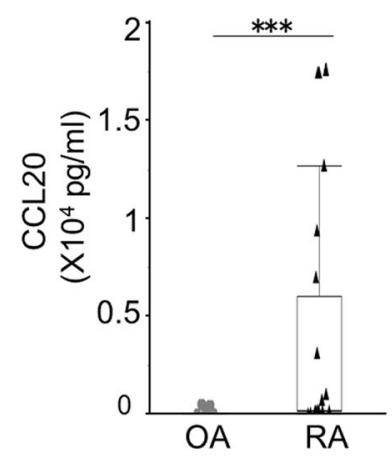

B

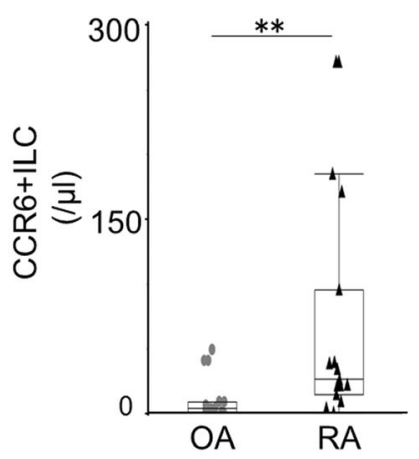

C

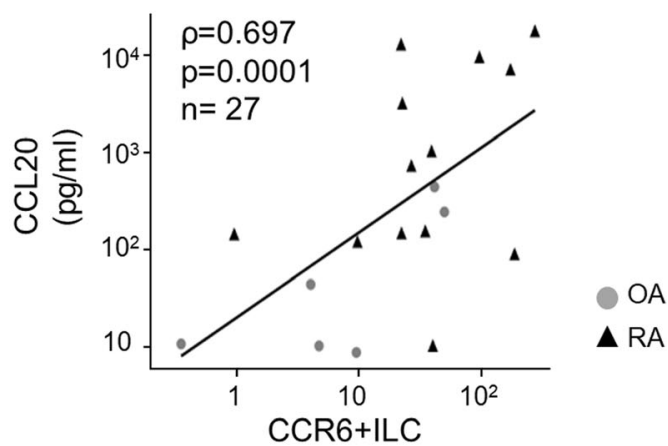

$(/ \mu l)$

Fig. 5 CCR6+ ILC number is positively correlated with SF CCL20 levels. a Comparison of CCL20 concentration in SF of patients with OA $(n=24)$ and RA $(n=16)$. CCL20 concentration in SF of patients with RA was significantly higher than that in SF from OA patients. CCL20 levels in synovial fluid were measured by ELISA. Data was analyzed using the Wilcoxon rank-sum test. $\left({ }^{*} p<0.05,{ }^{* *} p<0.01,{ }^{* *} p<0.001\right)$. b The absolute number of CCR6+ ILCS in SF of patients of OA $(n=13)$ and RA $(n=15)$. Data was analyzed using the Wilcoxon rank-sum test. $\left({ }^{*} p<0.05,{ }^{* *} p<0.01,{ }^{* * *} p<\right.$ 0.001). c Correlation of the number of CCR6+ ILCs and CCL20 concentration in SF of patients with OA $(n=13)$ and RA $(n=15)$. The number of CCR6+ ILCs was positively correlated with CCL20 concentration. Data are presented as scatter plots with a linear fit and analyzed by calculating Spearman's rank correlation coefficient 
clinical improvements [26, 27], while meta-analyses show favorable results for ACR20 or ACR50 improvement $[28,29]$. These results indicate that the relative importance of Th17 pathways differs among patients or at different disease stages. Accordingly, it is crucial to know which patients with RA will respond well to IL-17 blockade therapy. Identification of a surrogate marker for a high proportion of CCR6+ ILCs in SF might help stratify RA patients as candidates for anti-IL-17 therapy.

The limitation of our study was that we could not evaluate the correlations between CCR6+ ILCs and composite measures of RA disease activity, such as Disease Activity Score 28 (DAS28)-CRP or DAS28-ESR, due to unavailability of appropriate data. Secondly, the sample size of both mice and human studies was small. Lastly, we could not clearly identify ILCs in the synovium, the site of inflammation in RA. Though we detected ILC subsets in the synovium obtained from two patients with RA by flow cytometry (Additional file 6: Figure S6), the data was unsuitable to discuss because the sample size was too small. We also tried to identify ILCs in the joints of wild-type or CIA mice by immunohistochemistry, but we failed presumably due to technical problems. Recently, Noort et al. showed that ILC3s, which were defined as CD3- RORyt+ cells, were present in human synovium by immunohistochemistry [30]. Zhang et al. reported that recent advances at single-cell analysis, such as mass cytometry and RNA sequencing, contributed to identify new cell population in synovium that might be related to the pathogenesis of RA [31]. To provide more information about the role of ILCs in RA development, we should collect a large number of samples and evaluate ILCs in synovium by these new techniques.

In conclusion, the present study demonstrates that CCR6+ ILC3s are enriched in the inflamed joints of CIA mice and active RA patients and could play an important role in RA development.

\section{Additional files}

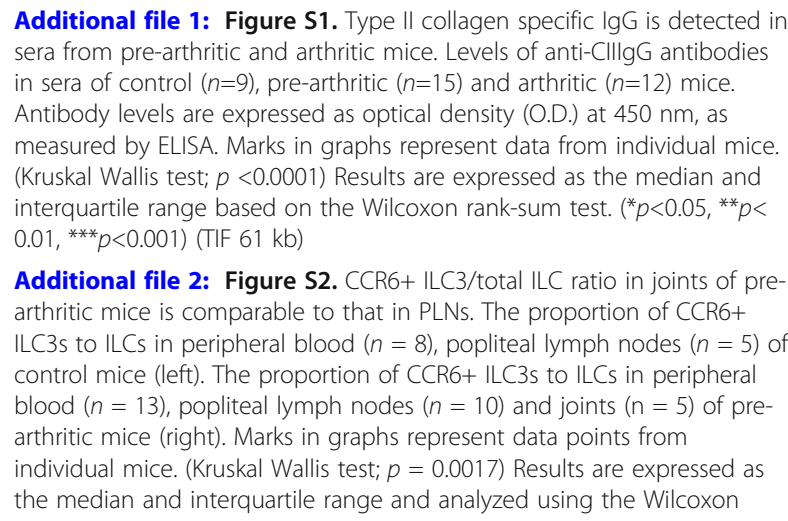

Additional file 2: Figure S2. CCR6+ ILC3/total ILC ratio in joints of prearthritic mice is comparable to that in PLNs. The proportion of CCR6+ ILC3s to ILCS in peripheral blood $(n=8)$, popliteal lymph nodes $(n=5)$ of control mice (left). The proportion of CCR6+ ILC3s to ILCs in peripheral blood ( $n=13)$, popliteal lymph nodes $(n=10)$ and joints $(n=5)$ of prearthritic mice (right). Marks in graphs represent data points from individual mice. (Kruskal Wallis test; $p=0.0017$ ) Results are expressed as the median and interquartile range and analyzed using the Wilcoxon

rank-sum test. $\left.{ }^{*} p<0.05,{ }^{* *} p<0.01,{ }^{* * *} p<0.001\right)$. N.S., not significant; N.D., not detected. (TIF $65 \mathrm{~kb}$ )

Additional file 3: Figure S3. ILC subsets in SF of RA patients. A. Gating strategy for ILCs from SF of a patient with RA. ILCs were defined as CD45+, lineage -, CD127+ cells. ILC1 cells: CRTH2- CD117- NKp44-; ILC2 cells: CRTH2+; NKp44- ILC3 cells: CRTH2-CD117+ NKp44-; and NKp44+ ILC3 cells: CRTH2- CD117+ NKp44+ (delete). B. Representative FACS plots of CCR6+ and NKp44+ ILCS from SF of patients with RA. These are gated on ILCS shown at left in panel A. (TIF $128 \mathrm{~kb}$ )

Additional file 4: Figure S4. Expression of RORC and Th17 cytokines mRNA in human CCR6+ ILCs. A. CCR6+ ILCs in human peripheral blood expresses RORC in the steady state. Results are shown as means \pm SD in triplicate samples of four patients. B. CCR6+ ILCs in human peripheral blood expresses IL-17A and IL-22 mRNA after stimulation with PMA/ ionomycin. Results are shown as means \pm SD in triplicate samples of two patients. (TIF $79 \mathrm{~kb}$ )

Additional file 5: Figure S5. Production of Th17 cytokines in human CCR6+ ILCs and Th subsets. A.B. CCR6+ ILCs in human peripheral blood produce Th17 cytokines after stimulation with PMA/ ionomycin for five hours. Representative data of the production of IL-17 (A) and IL-22 (B) by CCR6+ ILCS. C.D. Representative data of the production of IL-17 (C) and IL-22 (D) by Th1, Th2, Th17 and Th1/17 after stimulation with PMA/ ionomycin for five hours. Th subsets were defined as single cells within the lymphocyte gate on the scatter plot that were CD3 and CD4 positive. Th1, Th2, Th17 and Th1/17 were defined as CXCR3+ CCR6-, CXCR3CCR6-, CXCR3- CCR6+ and CXCR3+ CCR6+ respectively. (TIF 369 kb)

Additional file 6: Figure S6. ILC subsets in synovium of patients with RA. FACS plots of ILC subsets in synovium of patients with RA. (TIF 189 $\mathrm{kb})$

Additional file 7: Table S1. List of antibodies used in flow cytometry (DOCX $23 \mathrm{~kb}$ )

Additional file 8: Table S2. Clinical characteristics of patients with RA. TCZ, Tocilizumab. ETN, Etanercept. MZR, Mizoribine. IGU, Iguratimod. (DOCX $16 \mathrm{~kb})$

Additional file 9: Table S3. Correlation of clinical parameters with the proportion of ILC subsets in total ILCs based on the Spearman's rank correlation coefficient. $\left({ }^{*} p<0.05,{ }^{* *} p<0.01,{ }^{* * *} p<0.001\right)$. (TIF $\left.192 \mathrm{~kb}\right)$

\section{Abbreviations}

ILCs: Innate lymphoid cells; RA: Rheumatoid arthritis; CIA: Collagen-induced arthritis; PB: Peripheral blood; LNs: Lymph nodes; SF: Synovial fluid; TJC: Tender joint count; SJC: Swollen joint count; CCL20: CC chemokine ligand 20; T-bet: T-box transcription factor; IFN- $\gamma$ : Interferon- $\gamma$; GATA3: GATAbinding protein 3; IL-4: Interleukin-4; RORyt: Retinoic acid receptor-related orphan receptor yt; CCR6: CC chemokine receptor 6; NCR: Natural cytotoxicity receptor; NK: Natural killer; SpA: Spondyloarthritis; Cll: Type II collagen; CFA: Complete Freund's adjuvant; ELISA: Enzyme-linked immunosorbent assay; OA: Osteoarthritis; CRTH2: Chemoattractant receptor homologous molecule expressed on Th2 cells; TFs: Transcription factors; Tbx21: T-box 21; RORC: RAR-related orphan receptor C; PLNs: Popliteal lymph nodes; CRP: C-reactive protein; ESR: Erythrocyte sedimentation rate; RF: Rheumatoid factor; DAS28: Disease Activity Score 28

\section{Acknowledgements}

We are grateful to Drs. Shun-ichiro Ota, Takeshi Harada, and Keisuke Kashiwado for their contributions to the data acquisition and Elise Lamar for proofreading the manuscript.

\section{Authors' contributions}

All authors drafted the article and revised it critically for important intellectual content, and all authors approved the final manuscript.

\section{Funding}

This work was supported by a Grant-in-Aid for Scientific Research(C) and a grant from the Japan Rheumatism Foundation. 


\section{Availability of data and materials}

All data generated or analyzed during this study are included in this published article and its supplementary information files.

\section{Ethics approval and consent to participate}

All animal experimental procedures were approved by the Committee of Ethics on Animal Experiment at the Faculty of Medicine, Kyushu University. Experiments were conducted under the control of the Guidelines for Animal Experimentation at the Faculty of Medicine, Kyushu University. Informed consent was obtained from all subjects in accordance with the Declaration of Helsinki. The Institutional Review Board of Kyushu University Hospital approved all research on human subjects.

\section{Consent for publication}

All patients provided written informed consent for the publication of information resulting from the trial without any personally identifying information.

\section{Competing interests}

The authors declare that they have no competing interests.

\section{Author details}

'Department of Medicine and Biosystemic Science, Kyushu University Graduate School of Medical Sciences, 3-1-1 Maidashi, Higashi-ku, Fukuoka 812-8582, Japan. 'Division of Host Defense, Medical Institute of Bioregulation, Kyushu University, Fukuoka, Japan. ${ }^{3}$ Department of Stem Cell Biology and Medicine Faculty of Medical Sciences, Kyushu University, Fukuoka, Japan. ${ }^{4}$ Department of Internal Medicine, Kyushu University Beppu Hospital, Beppu, Japan. ${ }^{5}$ Faculty of Medical Sciences Medical Education, Kyushu University, Fukuoka, Japan.

Received: 26 March 2019 Accepted: 19 August 2019 Published online: 30 August 2019

\section{References}

1. Spits H, Artis D, Colonna M, Diefenbach A, Di Santo JP, Eberl G, et al. Innate lymphoid cells--a proposal for uniform nomenclature. Nat Rev Immunol. 2013;13:145-9.

2. Artis $\mathrm{D}$, Spits $\mathrm{H}$. The biology of innate lymphoid cells. Nature. 2015; 517:293-301.

3. Pazina T, Shemesh A, Brusilovsky M, Porgador A, Campbell K. Regulation of the functions of natural cytotoxicity receptors by interactions with diverse ligands and alterations in splice variant expression. Front Immunol. 2017;30(8):369.

4. Shikhagaie M, Germar K, Bal S, Ros X, Spits H. Innate lymphoid cells in autoimmunity: emerging regulators in rheumatic diseases. Nat Rev Rheumatol. 2017:13:164.

5. Wenink M, Leijten E, Cupedo T, Radstake T. Review: innate lymphoid cells: sparking inflammatory rheumatic disease? Arthritis Rheumatol. 2017;69:885-97.

6. Ebbo M, Crinier A, Vély F, Vivier E. Innate lymphoid cells: major players in inflammatory diseases. Nat Rev Immunol. 2017;17:665-78.

7. Leijten E, Kempen T, Boes M, Amelsfort J, Hijnen D, Hartgring S, et al. Brief report: enrichment of activated group 3 innate lymphoid cells in psoriatic arthritis synovial fluid. Arthritis Rheumatology. 2015;67:2673-8.

8. Ciccia F, Guggino G, Rizzo A, Saieva L, Peralta S, Giardina A, et al. Type 3 innate lymphoid cells producing $\mathrm{LL}-17$ and IL-22 are expanded in the gut, in the peripheral blood, synovial fluid and bone marrow of patients with ankylosing spondylitis. Ann Rheum Dis. 2015;74:1739-47.

9. Soare A, Weber S, Maul L, Rauber S, Gheorghiu A, Luber M, et al. Cutting edge: homeostasis of innate lymphoid cells is imbalanced in psoriatic arthritis. J Immunol. 2018;200:1249-54.

10. Blijdorp I, Menegatti S, Mens L, Sande M, Chen S, Hreggvidsdottir H, et al. Expansion of interleukin-22- and granulocyte-macrophage colonystimulating factor-expressing, but not interleukin-17A-expressing, group 3 innate lymphoid cells in the inflamed joints of patients with spondyloarthritis. Arthritis Rheumatol. 2019;71:392-402.

11. Rauber S, Luber M, Weber S, Maul L, Soare A, Wohlfahrt T, et al. Resolution of inflammation by interleukin-9-producing type 2 innate lymphoid cells. Nat Med. 2017;23:938-44.
12. Omata Y, Frech M, Primbs T, Lucas S, Andreev D, Scholtysek C, et al. Group 2 innate lymphoid cells attenuate inflammatory arthritis and protect from bone destruction in mice. Cell Rep. 2018;24:169-80.

13. Hirota $K$, Hashimoto $M$, Ito $Y$, Matsuura $M$, Ito $H$, Tanaka $M$, et al. Autoimmune Th17 cells induced synovial stromal and innate lymphoid cell secretion of the cytokine GM-CSF to initiate and augment autoimmune arthritis. Immunity. 2018;48:1220-32.

14. Dalbeth N, Callan M. A subset of natural killer cells is greatly expanded within inflamed joints. Arthritis Rheumatism. 2002;46:1763-72.

15. Ren J, Feng Z, Lv Z, Chen X, Li J. Natural killer-22 cells in the synovial fluid of patients with rheumatoid arthritis are an innate source of interleukin 22 and tumor necrosis factor-a. J Rheumatol. 2011;38:2112-8.

16. Zhu J, Jia E, Zhou Y, Xu J, Feng Z, Wang H, et al. Interleukin-22 secreted by NKp44+ natural killer cells promotes proliferation of fibroblast-like synoviocytes in rheumatoid arthritis. Medicine. 2015;94:e2137.

17. Roeleveld D, Koenders M. The role of the Th17 cytokines IL-17 and IL-22 in immunotherapy. Cytokine. 2015;74:101-7.

18. Kochi Y, Okada Y, Suzuki A, Ikari K, Terao C, Takahashi A, et al. A regulatory variant in CCR6 is associated with rheumatoid arthritis susceptibility. Nat Genet. 2010;42:515-9.

19. Walker J, Barlow J, McKenzie A. Innate lymphoid cells-how did we miss them? Nat Rev Immunol. 2013;13:75-87.

20. Chiossone L, Chaix J, Fuseri N, Roth C, Vivier E, Walzer T. Maturation of mouse NK cells is a 4-stage developmental program. Blood. 2009;113:5488-96.

21. Hazenberg M, Spits H. Human innate lymphoid cells. Blood. 2014;124:700-9.

22. Lee AY, Körner H. CCR6 and CCL20: emerging players in the pathogenesis of rheumatoid arthritis. Immunol Cell Biol. 2014;92:354-8.

23. Hirota K, Yoshitomi H, Hashimoto M, Maeda S, Teradaira S, Sugimoto N, et al. Preferential recruitment of CCR6-expressing Th17 cells to inflamed joints via CCL20 in rheumatoid arthritis and its animal model. J Exp Medicine. 2007;204:2803-12.

24. Rodríguez-Carrio J, Hähnlein J, Ramwadhdoebe T, Semmelink J, Choi I, van Lienden $\mathrm{K}$, et al. Brief report: altered innate lymphoid cell subsets in human lymph node biopsy specimens obtained during the at-risk and earliest phases of rheumatoid arthritis. Arthritis Rheumatol. 2017:69:70-6.

25. Lubberts E. IL-17/Th17 targeting: on the road to prevent chronic destructive arthritis? Cytokine. 2008;41:84-91.

26. Martin D, Churchill M, Flores-Suarez L, Cardiel M, Wallace D, Martin R, et al. A phase Ib multiple ascending dose study evaluating safety, pharmacokinetics, and early clinical response of brodalumab, a human anti -IL-17R antibody, in methotrexate-resistant rheumatoid arthritis. Arthritis Res Ther. 2013;15:1-9.

27. Genovese M, Durez P, Richards H, Supronik J, Dokoupilova E, Mazurov V, et al. Efficacy and safety of secukinumab in patients with rheumatoid arthritis: a phase II, dose-finding, double-blind, randomised, placebo controlled study. Ann Rheum Dis. 2013;72:863-9.

28. Wei M, Dian D. Efficacy and safety of monoclonal antibodies targeting interleukin-17 pathway for inflammatory arthritis: a meta-analysis of randomized controlled clinical trials. Drug Des Devel Ther. 2016;10:2771-7.

29. Kunwar S, Dahal K, Sharma S. Anti-IL-17 therapy in treatment of rheumatoid arthritis: a systematic literature review and meta-analysis of randomized controlled trials. Rheumatol Int. 2016;36:1065-75.

30. Noort AR, van Zoest KP, van Baarsen LG, Maracle CX, Helder B, Papazian N, et al. Tertiary lymphoid structures in rheumatoid arthritis: NF-KB-inducing kinase-positive endothelial cells as central players. Am J Pathol. 2015;185:1935-43.

31. Zhang F, Wei K, Slowikowski K, Fonseka CY, Rao DA, Kelly S, et al. Defining inflammatory cell states in rheumatoid arthritis joint synovial tissues by integrating single-cell transcriptomics and mass cytometry. Nat Immunol. 2019;20:928-42.

\section{Publisher's Note}

Springer Nature remains neutral with regard to jurisdictional claims in published maps and institutional affiliations. 